\title{
Optimal Symmetric Double-sided Signal Clipping in DCO-OFDM Visible Light Communication
}

\author{
Meishuang Yu, Zaichen Zhang, Liang Wu and Jian Dang \\ National Mobile Communications Research Laboratory \\ Southeast University, Nanjing, China \\ Email: \{yumeishuang, zczhang, wuliang,newwanda\}@seu.edu.cn
}

\begin{abstract}
In this paper, the optimal DC bias value of symmetric double-sided signal clipping in DC-biased optical orthogonal frequency division multiplexing (DCO-OFDM) visible light communication (VLC) system is deduced. Double-sided clipping is necessary due to the unipolarity of the optical intensity-modulated, direct-detected (IM/DD) system and power limit of the transmitter. Symmetric double-sided clipping is adopted to achieve wider dynamic range. Clipping noise is analyzed for system performance investigation. The optimal DC bias value is deduced by minimizing the system bit error ratio (BER) at given transmitter optical power. An equation of signal to noise ratio (SNR), signal energy, and optimal DC bias value is derived. Finally, the optimal algorithm is verified through a BER simulation at different DC bias values.

Index Terms-Double-sided clipping; DCO-OFDM; VLC
\end{abstract}

\section{INTRODUCTION}

Light emitting diode (LED) has been more and more popular in illumination system due to its advantages of high light, long operation life and high power efficiency. The available modulation bandwidth of LED lies in $\mathrm{MHz}$ [1]. When properly modulated, visible light communication (VLC) system can achieve very high transmission speed up to Gbit/s [2-3], which makes it possible for LED to serve for lighting and communication simultaneously. Compared with traditional wireless communication, VLC has a lot of merits including license free spectrum, large potential capacity, no electromagnetic interference, and high security.

Orthogonal frequency division multiplexing (OFDM) is one of the main modulation techniques to implement high speed VLC system. The optical intensity-modulated, direct-detected (IM/DD) system can only transmit real non-negative signal, while the traditional baseband OFDM symbol is generally bipolar and complex. To deal with this problem, DC-biased optical OFDM (DCO-OFDM) [4],

This work is supported by 863 project (No. 2013AA013601), NSFC project (No. 61223001), Jiangsu NSF project (No. BK20140646), the Research Fund of NCRL (No. 2014A03, 2014B03, and 2014B04), the Research Fund of ZTE Corporation, the Fundamental Research Funds of the Central Universities (No. 2242014K40033), and the PRPFN of Jiangsu FNII (No. BY2013095-1-18). asymmetrically clipped optical OFDM (ACO-OFDM) [5], and many other improved modulation techniques are introduced to VLC system. To get a real signal at the OFDM output, the data before modulation must be ensured to be Hermitian symmetry. DCO-OFDM adds a DC bias to make the signal non-negative, while ACO-OFDM only transmits the non-negative part of the signal and ignores the negative part. DCO-OFDM has higher bandwidth efficiency than ACO-OFDM, while ACO-OFDM has higher power efficiency [6]. In VLC system, one of the limits of achieving high speed is the LED's narrow bandwidth. Meanwhile, compared with ACO-OFDM, DCO-OFDM is easier to implement. In this paper, main attention is paid to the clipping in DCO-OFDM.

A lot of researches about OFDM clipping are already available. Many of them are single-sided [7-8], that is only the negative part of the signal is clipped and the possible extremely high amplitude is ignored. Generally, the transmitter has both average and instantaneous maximum power restrictions. The high peak to average ratio (PAR) of OFDM [9] determines that double-sided clipping is necessary. Double-sided clipping in ACO-OFDM has already been considered in [10]. This paper focuses on the double-sided clipping in DCO-OFDM. Too much clipping may cause unnecessary signal distortion under certain conditions, while too less clipping increases the demands for transmitter amplifier and digital-to-analog converter (DAC). In this paper, the effects of symmetric double-sided clipping are investigated, and clipping noise is analyzed. The optimal DC bias value is deduced by minimizing the system BER at a given transmitter power. The result is verified through simulation with different DC bias values.

The reminder of this paper is organized as follows : DCO-OFDM system and double-sided clipping are introduced in Section II. In Section III, the analysis of double-sided clipping is given and the optimal clipping value is deduced. In Section IV, simulation results are presented. Finally, Section V draws the conclusions.

\section{SYSTEM MODEL}

This section focuses on the traditional DCO-OFDM system model. Fig. 1 shows a typical IM/DD DCO-OFDM system. The system is composed of transmitter with LED and receiver with photodiode (PD). At the transmitter, a high-speed data stream is split into multiple low-speed data steams through a serial-to-parallel (S/P) converter, and then mapped to the $\mathrm{M}$-ary quadrature amplitude modulation (M-QAM) constellation. As the LED can only carry real signal, data are 
constrained to have Hermitian symmetry before the inverse fast Fourier transform (IFFT). $X_{k}$ is the complex symbol on the k-th subcarrier in frequency domain :

$$
X_{k}=X_{k}^{I}+j X_{k}^{Q}, \quad k=0,1, \ldots, N-1
$$

where $X_{k}^{I}$ and $X_{k}^{Q}$ represent the real and imaginary component of the symbol, respectively. $\mathrm{N}$ is the number of the subcarriers, as well as the FFT/IFFT size, and $\mathrm{j}$ is the imaginary unit. Hermitian symmetry is ensured by setting:

$$
\begin{aligned}
& X_{0}=X_{N / 2}=0 \quad \text { and } \\
& X_{k}=X_{N-k}^{*} \quad(1 \leq k \leq N / 2-1)
\end{aligned}
$$

IFFT transforms the frequency domain signal into time domain. The n-th time domain sample $x_{n}$ can be represented as below:

$$
\begin{aligned}
x_{n} & =\frac{1}{N} \sum_{k=0}^{N-1} X_{k} e^{j \frac{2 \pi}{N} k n} \\
& =\frac{1}{N} \sum_{k=0}^{N / 2-1}\left(X_{k} e^{j \frac{2 \pi}{N} k n}+X_{k}^{*} e^{-j \frac{2 \pi}{N} k n}\right) \\
& =\frac{2}{N} \sum_{k=0}^{N / 2-1}\left\{X_{k}^{I} \cos \left(\frac{2 \pi}{N} k n\right)-X_{k}^{Q} \sin \left(\frac{2 \pi}{N} k n\right)\right\}
\end{aligned}
$$

As (3) shows, $x_{n}$ is a real-valued signal, and it is the sum of multi independent identically distributed (i.i.d) signals. For large $\mathrm{N}$ values, $x_{n}$ is approximately Gaussian distributed due to the central-limit theorem [11].

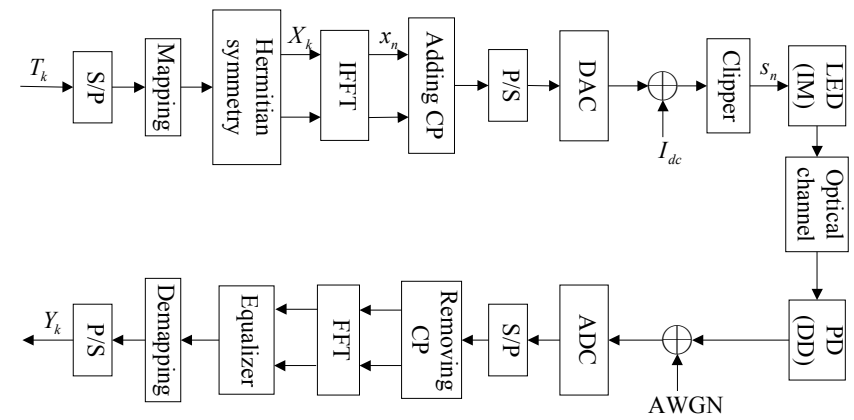

Fig. 1 DCO-OFDM system

To reduce or even eliminate inter-symbol interference (ISI) and inter-carrier interference (ICI) caused by delay spread or dispersion, a certain length of cyclic prefix (CP) is inserted into each OFDM symbol after IFFT. Then the data is sent to a DAC through a parallel-to-serial $(\mathrm{P} / \mathrm{S})$ converter. The DAC converts digital signal to analog signal, which is usually bipolar. Therefore, a DC bias $I_{d c}$ is added to make the signal unipolar. As mentioned above, symmetric double-sided clipping is adopted. We denote the symbol after clipping by $S_{n}$ :

$$
s_{n}=\left\{\begin{array}{lr}
0 & x_{n}<-I_{d c} \\
x_{n}+I_{d c} & -I_{d c} \leq x_{n} \leq I_{d c} \\
2 I_{d c} & x_{n}>I_{d c}
\end{array}\right.
$$

The symbol after clipping is passed to the LED driving circuit. The symbol is turned into optical signal and transmitted in the optical channel. The distorted signal is influenced by the thermal noise and shot noise [12] at the receiver circuit, which are modeled as additive white gaussian noise (AWGN). Performance of the clipped system is analyzed in the next section based on this assumption.

\section{SYSTEM PERFORMANCE ANALYSIS}

The symbol after IFFT, which may be further amplified, is clipped before being transmitted to the AWGN channel. Denoting the signal at the receiver by $r_{n}$, the clipping process can be taken as adding a clipping noise to the system, that is:

$$
r_{n}=x_{n}+e_{n, 1}+e_{n, 2}
$$

where $e_{n, 1}$ is the clipping noise and $e_{n, 2}$ is the AWGN, $e_{n, 2} \sim N\left(0, \sigma_{2}^{2}\right)$.

\section{A. Clipping Noise Analysis}

As shown in (4) and Fig. 2, the DC bias $I_{d c}$, is added to the OFDM symbol $x_{n}$, and clipped to obtain $s_{n} \cdot x_{n}$ is an zero mean Gaussian distributed variable with variance $\sigma_{0}^{2}$. Probability density function (PDF) of $x_{n}$ is:

$$
f_{x_{n}}(x)=\frac{1}{\sqrt{2 \pi} \sigma_{0}} e^{-\frac{x^{2}}{2 \sigma_{0}^{2}}}
$$

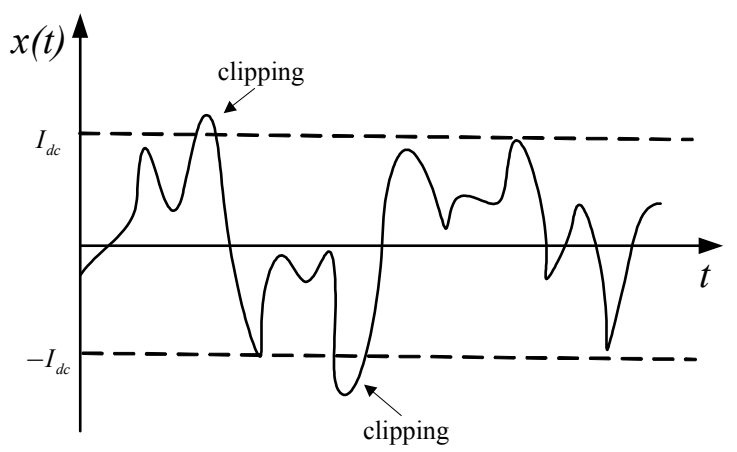

Fig. 2 the way to clip

When $x_{n}$ is in the range of $-I_{d c} \sim I_{d c}$, signal is not clipped, that is $e_{n, 1}=0$. The probability can be represented as:

$$
P_{e_{n, 1}}(0)=P\left(e_{n, 1}=0\right)=\int_{-I_{d c}}^{I_{d c}} f_{x_{n}}(x) d x
$$

When $x_{n}$ is beyond that range:

$$
\begin{gathered}
f_{e_{n, 1}}\left(x-I_{d c}\right)=f_{x_{n}}(x) \quad x>I_{d c} \\
f_{e_{n, 1}}\left(x+I_{d c}\right)=f_{x_{n}}(x) \quad x<-I_{d c}
\end{gathered}
$$

that is: 


$$
f_{e_{n, 1}}(x)= \begin{cases}f_{x_{n}}\left(x+I_{d c}\right) & x>0 \\ f_{x_{n}}\left(x-I_{d c}\right) & x<0\end{cases}
$$

For simplifying analysis, we appropriate $e_{n, 1}$ as AWGN. Its mean $m_{e_{n, 1}}$ and variance $\sigma_{1}^{2}$ are calculated as below:

$$
\begin{aligned}
& m_{e_{n, 1}}=\int_{-\infty}^{\infty} x * f_{e_{n, 1}}(x) d x=0 \\
\sigma_{1}^{2} & =\int_{-\infty}^{\infty} x^{2} f_{e_{n, 1}}(x) d x \\
& =2 \int_{0}^{\infty} \frac{x^{2}}{\sqrt{2 \pi} \sigma_{0}} e^{-\frac{\left(x+I_{d c}\right)^{2}}{2 \sigma_{0}^{2}}} d x \\
= & 2\left[\left(I_{d c}^{2}+\sigma_{0}^{2}\right) \Phi\left(-\frac{I_{d c}}{\sigma_{0}}\right)-\frac{\sigma_{0} I_{d c}}{\sqrt{2 \pi}} e^{-\frac{I_{d c}^{2}}{2 \sigma_{0}^{2}}}\right]
\end{aligned}
$$

where

$$
\Phi(x)=\int_{-\infty}^{x} \frac{1}{\sqrt{2 \pi}} e^{-\frac{\tau^{2}}{2}} d \tau
$$

That is $e_{n, 1} \sim N\left(0, \sigma_{1}^{2}\right)$, and variance $\sigma_{1}^{2}$ is shown in (12).

\section{B. Optimal Clipping Value}

The following analysis is carried out by minimizing system BER at a given transmitter power $P_{t}$ to get the optimal DC bias value.

When the clipping level is not high, the following equation is established:

$$
P_{t}=I_{d c}^{2}+\sigma_{0}^{2}
$$

For M-QAM in AWGN environment, its BER performance is [13]:

$$
\begin{gathered}
P_{e, M-Q A M}=1-\left(1-P_{e, \sqrt{M}-P A M}\right)^{2} \\
P_{e, \sqrt{M}-P A M}=2\left(1-\frac{1}{\sqrt{M}}\right) Q\left(\sqrt{\frac{3 \log _{2} M}{M-1} \frac{E_{\text {bavg }}}{N_{0}}}\right)
\end{gathered}
$$

$E_{\text {avg }}$ is the average energy of each bit, $N_{0}$ is the variance of the system noise. As (5) shows, $r_{n}$ can be represented as:

$$
r_{n}=x_{n}+e_{n, 1}+e_{n, 2}
$$

where $e_{n, 1} \sim N\left(0, \sigma_{1}^{2}\right), e_{n, 2} \sim N\left(0, \sigma_{2}^{2}\right)$. As $e_{n, 1}$ and $e_{n, 2}$ are i.i.d and Gaussian distributed variables, $N_{0}$ can be represented as:

$$
N_{0}=\sigma_{1}^{2}+\sigma_{2}^{2}
$$

To get the optimal $I_{d c}$, derivate $P_{e, M-Q A M}$ by $I_{d c}$ and set the result to zero:

$$
\frac{\partial P_{e, M-Q A M}}{\partial I_{d c}}=0
$$

which can be simplified as:

$$
\frac{2}{\sqrt{2 \pi}} \frac{\sigma_{0}^{3}}{I_{d c}^{3}} e^{-\frac{I_{c c}^{2}}{2 \sigma_{0}^{2}}}=\frac{2\left(I_{d c}^{2}+\sigma_{0}^{2}\right)}{I_{d c}^{2}} \Phi\left(-\frac{I_{d c}}{\sigma_{0}}\right)-\frac{2 \sigma_{0}}{\sqrt{2 \pi} D C} e^{-\frac{I_{d c}^{2}}{2 \sigma_{0}^{2}}}+\frac{\sigma_{2}^{2}}{I_{d c}^{2}}
$$

Signal to noise ratio (SNR) in electrical domain is defined as:

$$
S N R=\frac{P_{t}}{\sigma_{2}^{2}}
$$

where $P_{t}$ is defined by (14). Define a coefficient $t$,

$$
t=\frac{\sigma_{0}}{I_{d c}}
$$

and substitute $t$ and SNR into (20), we can get:

$$
\frac{2}{\sqrt{2 \pi}} t e^{-\frac{1}{2 t^{2}}}-2 \Phi\left(-\frac{1}{t}\right)-\frac{1}{S N R}=0
$$

It is an equation about SNR and $t$, it shows that $t$ will only be affected by the value of SNR. As $P_{t}$ is assumed to be constant, we can get the optimal $I_{d c}$ :

$$
I_{d c}=\sqrt{\frac{P_{t}}{1+t^{2}}}
$$

\section{Simulation Results}

According to (23), optimal coefficient $t$ at different SNR is given in Fig. 3, where $t$ is defined by (22). The figure shows that as the SNR increases, the value of $t$ gets smaller. It means that for a given $P_{t}$, the optimal DC bias is higher at higher SNR. In other words, clipping level is smaller at higher SNR. As mentioned above, (14) is accurate only under the assumption of small clipping level. Therefore, the algorithm is more accurate at higher SNR.

Fig. 4 gives the BER performances of different DC bias values in 4-QAM DCO-OFDM. When the DC bias value is $\sqrt{E\left\{x^{2}\right\}}=\sigma_{0}, 84.13 \%$ of $x_{n}$ is non-negative. When the DC bias value is $2 \sigma_{0}, 97.72 \%$ of $x_{n}$ is non-negative, which means that there is almost no clipping. Compared with the above two mentioned values, optimal DC bias value shows the best performance. We can find in Fig. 3 that coefficient $t \leq 2$ when $S N R \geq 1 d B$, which means that the optimal DC bias is always smaller than $2 \sigma_{0}$. When $B E R=2 * 10^{-3}$, which is within the forward error correction (FEC) limit, the optimal $I_{d c}$ has a $1.2 \mathrm{~dB}$ merit compared with $I_{d c}=2 \sigma_{0}$. The advantage is more obvious at higher SNR. This also shows that the algorithm is more accurate at higher SNR.

\section{CONCLUSION}

This paper gives the analysis of the clipping noise caused by the symmetric double-sided clipping in DCO-OFDM, and deduces the optimal clipping value at a given transmitter optical power by minimizing the system BER in AWGN channel. An equation about signal energy, DC bias value, and SNR is given. The performances of different clipping values are compared by simulations. Simulation results show that the 
algorithm proposed is effective. The algorithm is more accurate and performs better at higher SNR.

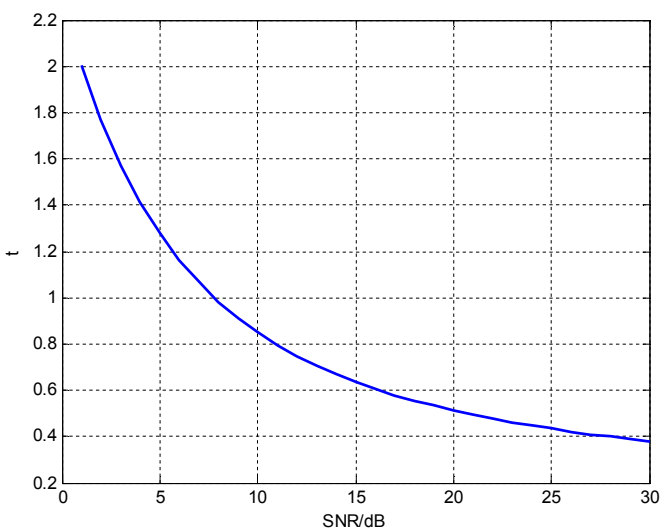

Fig. 3 t vs. SNR

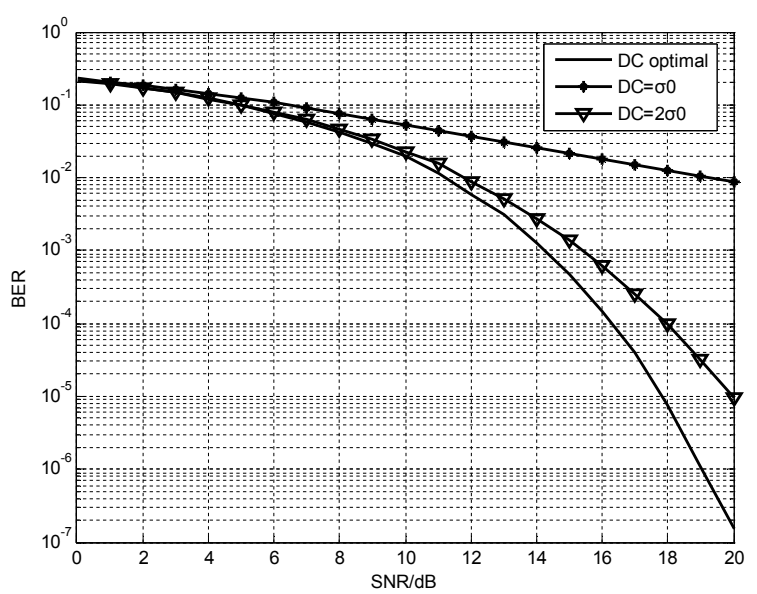

Fig. 4 BER at different DC bias values

\section{REFERENCES}

[1] Grubor, Jelena, et al. "Wireless high-speed data transmission with phosphorescent white-light LEDs." ECOC 2007 (2007).

[2] Cossu, G., et al. "3.4 Gbit/s visible optical wireless transmission based on RGB LED." Optics express 20.26 (2012): B501-B506.

[3] Azhar, Ahmad Helmi, T. Tran, and Dominic O'Brien. "A Gigabit/s indoor wireless transmission using MIMO-OFDM visible-light communications." Photonics Technology Letters, IEEE 25.2 (2013): 171-174.

[4] Carruthers, Jeffrey B., and Joseph M. Kahn. "Multiple-subcarrier modulation for nondirected wireless infrared communication." Selected Areas in Communications, IEEE Journal on 14.3 (1996): 538-546.

[5] Armstrong, Jean, and A. J. Lowery. "Power efficient optical OFDM."Electronics Letters 42.6 (2006): 370-372.

[6] Armstrong, Jean, and Brendon Schmidt. "Comparison of asymmetrically clipped optical OFDM and DC-biased optical OFDM in AWGN."Communications Letters, IEEE 12.5 (2008): 343-345.

[7] Ochiai, Hideki, and Hideki Imai. "Performance analysis of deliberately clipped OFDM signals." Communications, IEEE Transactions on 50.1 (2002): 89-101.

[8] Chen, Liang, Brian Krongold, and Jamie Evans. "Performance analysis for optical OFDM transmission in short-range IM/DD systems." Journal of Lightwave Technology 30.7 (2012): 974-983.

[9] KHAN, MOHSIN, SAMIMA IQBAL, and WASEEM ASGHAR. "A Review Paper On: The Papr Analysis Of Orthogonal Frequency Division Multiplexing (OFDM)." International journal (2014).

[10] Dimitrov, Svilen, Sinan Sinanovic, and Harald Haas. "Double-sided signal clipping in ACO-OFDM wireless communication systems." Communications (ICC), 2011 IEEE International Conference on. IEEE, 2011.

[11] Feller, Willliam. An introduction to probability theory and its applications. Vol. 2. John Wiley \& Sons, 2008.

[12] Kahn, Joseph M., and John R. Barry. "Wireless infrared communications."Proceedings of the IEEE 85.2 (1997): 265-298.

[13] John G. Proakis, Masoud Salehi. "Digital Communications." 5th ed. New York: McGraw-Hill, 2008, pp. 198 